\title{
Intestinal alkaline phosphatase in the diagnosis of liver disease
}

\author{
T. W. WARNES, P. HINE, AND G. KAY \\ From the Department of Gastroenterology, Manchester Royal Infirmary, Manchester
}

SUMMARY Alkaline phosphatase isoenzymes have been studied by acrylamide gel disc electrophoresis in 76 patients with liver disorders comprising 15 with an extrahepatic lesion, 53 with an intrahepatic lesion, and eight patients who had features of both intra- and extrahepatic disease. No intestinal band was found in the 15 cases of extrahepatic liver disease, in marked contrast to the patients with intrahepatic lesions in whom an intestinal band was found in $45 \%$ of cases. The intrahepatic group was heterogeneous, a high incidence of intestinal bands being found in patients with cirrhosis of the liver. It is concluded that where a raised serum alkaline phosphatase is found in a patient with jaundice, and a gut band is present on electrophoresis, the lesion is likely to be intrahepatic.

There is an urgent need for a simple and noninvasive test of liver function which will help to differentiate between a surgical case of jaundice requiring early intervention and a medical cause in which injudicious surgery may worsen the prognosis.

The association between high levels of serum alkaline phosphatase (AP) and liver disease has been known for a long time. For many years it was considered that a level of $30 \mathrm{KA}$ units/dl or greater implied obstructive jaundice, and values less than this an intrahepatic disorder (Roberts, 1933). However, since the introduction of the concept of intrahepatic cholestasis, it has been appreciated that the distinction between 'obstructive' and hepatocellular jaundice on the basis of quantitative estimation of serum AP is often impossible (Schimmel, 1968).

The recent development of reliable electrophoretic techniques has made possible the differentiation of raised levels of serum AP in bone and liver disease (Warnes, 1972). On acrylamide gel disc electrophoresis main, origin, and intestinal bands may be seen. The origin band is fairly specific for liver disease, being found in $78 \%$ of cases (Warnes et al., 1976). It has been shown that some patients with cirrhosis of the liver are characterised by increased amounts of intestinal AP in serum (Fishman et al., 1965). The present study is designed to see what diagnostic help the newer techniques of isoenzyme

Received for publication 26 November 1976 analysis offer in the diagnosis of liver disease, and, in particular, whether they are able to help in differentiating an intrahepatic from an extrahepatic cause of jaundice.

\section{Methods}

Seventy-six patients with liver disease were studied. Diagnoses were based on clinical findings, on laboratory tests of liver function which were supplemented by standard serological tests of autoimmune liver disease including antinuclear factor, LE cells, and antimitochondrial and smooth muscle antibodies, and on liver and pancreatic scans supplemented in most cases by liver biopsy, laparotomy, or necropsy findings. Oral cholecystography and intravenous cholangiography were performed in all cases where clinically indicated; an oral cholecystogram was not attempted if the serum bilirubin was above $2 \mathrm{mg} / \mathrm{dl}$ and an intravenous cholangiogram if the serum bilirubin exceeded $4 \mathrm{mg} / \mathrm{dl}$. The diagnoses thus reached are shown in Table 1: patients were then classified into those with an extrahepatic lesion (15), those with an intrahepatic lesion (53), and those who had features of both intra- and extrahepatic disease (eight).

Serum from these patients was subjected to electrophoresis in a Shandon electrophoresis tank using a $5 \%$ polyacrylamide gel and a continuous buffer system (tris-borate, pH 9.5). Enzyme bands were located by a diazo-cnupling technique using Fast 
Table 1 Classification of 76 patients with liver disease

\begin{tabular}{lc}
\hline Diagnosis & No. \\
\hline Extrahepatic (15) & \\
Carcinoma head of pancreas & 8 \\
Carcinoma gall bladder & 1 \\
Carcinoma extrahepatic ducts & 2 \\
Stone in common bile duct & 4 \\
Intrahepatic (53) & 3 \\
Primary liver carcinoma & 10 \\
Secondary liver carcinoma & 6 \\
Cirrhosis of liver & 9 \\
Primary biliary cirrhosis & 8 \\
Hepatitis & 3 \\
Infiltrations & 8 \\
Intrahepatic cholestasis & 6 \\
Other* & \\
Mixed (8) & 4 \\
Cholangiohepatitis & 4 \\
Miscellaneous $\dagger$ & \\
\hline
\end{tabular}

* Other comprised hepatic abscess (one), Budd-Chiari sundrome (one) passive venous congestion (two), rheumatoid arthritis with phenylbutazone toxicity (one) and diabetes with probable infective hepatitis (one)

† Miscellaneous comprised carcinoma common bile duct with secondary biliary cirrhosis and hepatic abscesses (one), carcinoma of lung in an alcoholic with probable cirrhosis and metastatic malignancy (one), fatty infiltration of liver associated with chronic pancreatitis (one), fatty liver associated with stone in common bile duct (one)

Blue BB and $\beta$-naphthyl phosphate (Smith et al., 1968).

Alkaline phosphatase was measured by the KindKing modification of the King-Armstrong method (Kind and King, 1954) and bilirubin by the method of Powell (1944).

\section{Results}

On electrophoresis a liver type main band (Fig. 1) was seen in all 76 patients with liver disease, the vast majority of whom also had an origin band which is not present in normal subjects (Warnes et al., 1976).

There was no significant difference between the mean AP values of the intra- and extrahepatic groups, but at the time of investigation the extrahepatic group had a significantly greater degree of jaundice (Table 2). The bilirubin concentrations in the intrahepatic group ranged from $0 \cdot 1-14.5 \mathrm{mg} / \mathrm{dl}$ : 22 patients in this group had a bilirubin of $2 \mathrm{mg} / \mathrm{dl}$ or over and in 12 the bilirubin concentration was over $5 \mathrm{mg} / \mathrm{dl}$ (Fig. 2). Conversely, in the extrahepatic group, the range of bilirubin levels was from 0.3 to $23.0 \mathrm{mg} / \mathrm{dl}$ and in six of these the bilirubin concentration was less than $2 \mathrm{mg} / \mathrm{dl}$. An intestinal band was found in none of the 15 patients with an extrahepatic lesion, although it was present in $45 \%$ of the intrahepatic group, a difference which is highly significant.

The intrahepatic group appeared to be heterogeneous, since an intestinal band was seen in over

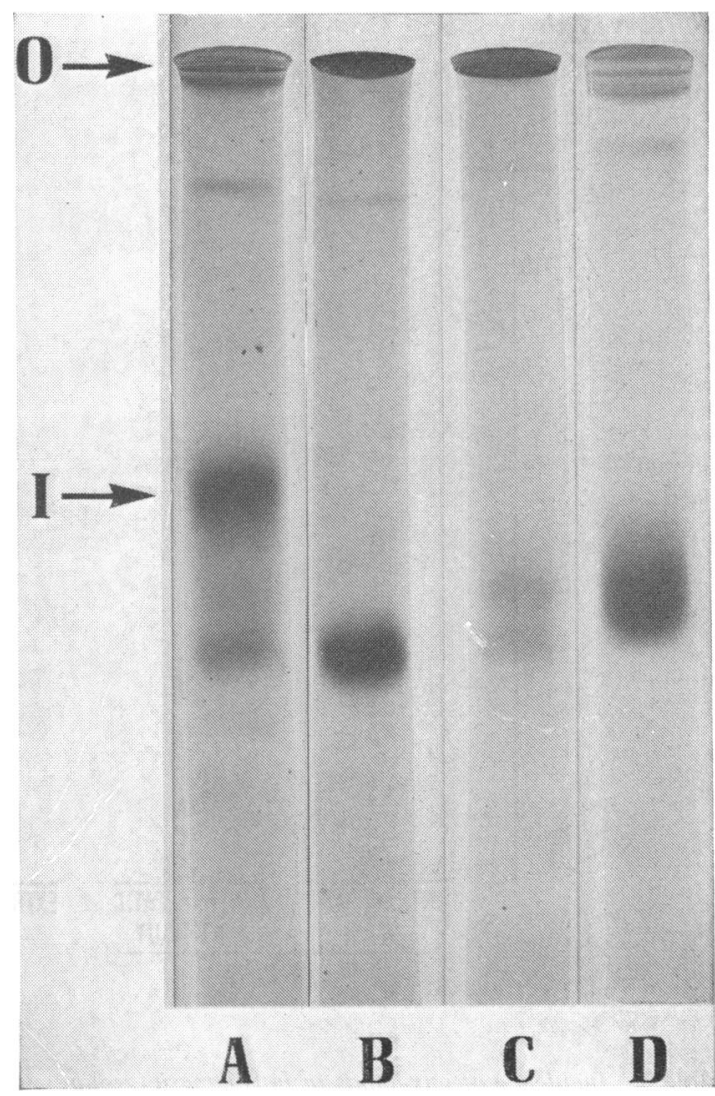

Fig. 1 Electrophoresis of liver, bone, and normal sera showing the origin band $(0)$, intestinal band $(I)$, and main bands.

A: intrahepatic liver disease. B: extrahepatic liver disease. $C$ : normal serum. D: bone disease.

Table 2 Comparison of alkaline phosphatase, bilirubin, and gut bands in intra-and extrahepatic liver disease

\begin{tabular}{|c|c|c|c|c|}
\hline & \multirow{2}{*}{$\begin{array}{l}\text { Mean } A P \\
(K . A U / d I)\end{array}$} & \multirow{2}{*}{$\begin{array}{l}\text { Bilirubin } \\
(m g / d l)\end{array}$} & \multicolumn{2}{|c|}{ Gut band } \\
\hline & & & no. & $\%$ \\
\hline Extrahepatic (15) & $\begin{array}{r}40.7 \\
\pm 26.9\end{array}$ & $\begin{array}{r}10.4 \\
\pm \quad 8.5\end{array}$ & 0 & 0 \\
\hline Intrahepatic (53) & $\begin{array}{r}33.9 \\
\pm 28.5\end{array}$ & $\begin{array}{r}2.9 \\
\pm \quad 3.6\end{array}$ & 24 & 45 \\
\hline$P$ (intra $v$ extra) & NS & $<0.001$ & $<0$. & \\
\hline
\end{tabular}

$\mathrm{AP}$ and bilirubin values are given as mean $\pm 1 \mathrm{SD}$.

$80 \%$ of cirrhotics, but in only $10 \%$ of cases of secondary cancer (Table 3). A test of heterogeneity applied to the intestinal bands in the various categories just failed to reach statistical significance $(P \bumpeq 0.06)$. 


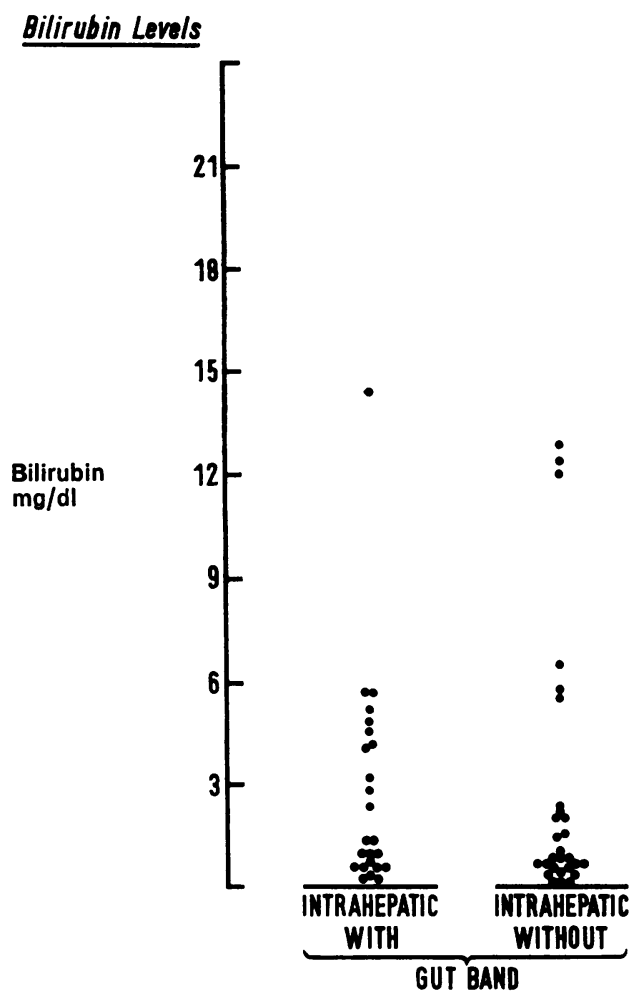

Fig. 2 Bilirubin levels in the intrahepatic and extrahepatic liver disease groups.
Table 3 Incidence of gut bands in intrahepatic group

\begin{tabular}{lcc}
\hline Diagnosis & \multicolumn{2}{l}{ Gut band } \\
\cline { 2 - 3 } & no. & $\%$ \\
\hline Cirrhosis (6) & 5 & 83 \\
Hepatitis (8) & 5 & 63 \\
Primary liver carcinoma (3) & 2 & 67 \\
Miscellaneous (6) & 4 & 67 \\
Primary biliary cirrhosis (9) & 3 & 33 \\
Intrahepatic cholestasis (8) & 2 & 25 \\
Infiltrations (3) & 2 & 67 \\
Secondary liver carcinoma (10) & 1 & 10 \\
Intrahepatic group (53) & 24 & 45 \\
Extrahepatic group (15) & 0 & 0 \\
Probability that intrahepatic group is truly & P $\bumpeq 0.06$ \\
heterogeneous & Cirrhcsis $\uparrow$ \\
& Secondary liver \\
& \multicolumn{2}{c}{ carcinoma $\downarrow$} \\
\hline
\end{tabular}

\section{Discussion}

In jaundiced patients the distinction between intrahepatic cholestasis and extrahepatic obstruction can be extremely difficult since the signs on clinical examination may be identical, and conventional liver function tests may be equivocal. In the presence of significant jaundice cholecystography and cholangiography frequently fail. Both techniques depend on the ability of the liver to excrete iodine-containing contrast materials; toxic reactions can occur. Oral cholecystography should not be undertaken when the serum conjugated bilirubin level is above $2 \mathrm{mg} / \mathrm{dl}$ and intravenous (or infusion) cholangiography when the serum bilirubin concentration exceeds $4 \mathrm{mg} / \mathrm{dl}$ (Sherlock, 1975). For this reason an oral cholecystogram was not obtained in 22 of the 53 patients in the intrahepatic group and in nine of the 15 patients in the extrahepatic group. Similarly, an intravenous or infusion cholangiogram was not obtained in 12 patients in the intrahepatic group and in nine patients in the extrahepatic group. In the deeply jaundiced patient with suspected extrahepatic obstruction liver biopsy is considered by most, though not all, clinicians to be contraindicated, and, even when a biopsy is obtained, in $10-15 \%$ of cases it is impossible to differentiate between intrahepatic cholestasis and extrahepatic obstruction (Williams, 1968). Percutaneous cholangiography has real dangers (Evans et al., 1962), though these have diminished considerably since the introduction of the 'fine needle' technique (Okuda et al., 1974). Failure to fill a duct does not completely exclude extrahepatic obstruction (James, 1971), and normal ducts may not be outlined. More recently it has been shown that in skilled hands endoscopic cannulation 
of the ampulla of Vater (ERCP) is of value in the difficult cases of jaundice. However, the technique is expensive and difficult to learn, and it fails to differentiate between intrahepatic and extrahepatic cholestasis in $20-30 \%$ of cases. Complications include cholangitis, pancreatitis, septicaemia, damage to the papilla and ducts, and the transmission of hepatitis. Deaths have been reported (Cotton, 1972).

The main significance of the present findings is based on the absence of a gut band in patients with an extrahepatic cause of jaundice. The explanation for this is uncertain. However, it is known that there is an association between the incidence of the occurrence of intestinal phosphatase in serum in health (Langman et al., 1966) with ABO blood group and secretor status, and in cirrhosis of the liver the occurrence of intestinal bands is also correlated with blood group (Stolbach et al., 1967). It is therefore possible that the difference we have found between the intrahepatic and extrahepatic groups could be related to differing $\mathrm{ABO}$ blood group distributions. It is also known that the appearance of the gut band in serum is dependent on the ingestion of fat (Warnes, 1972) and the observed difference between intra- and extrahepatic lesions could be partly due to malabsorption of fat in extrahepatic obstruction with a resultant failure of passage of brush border AP into lymph and thence into the general circulation. It has been shown in the experimental animal that complete obstruction of the common bile duct prevents release of AP into the intestinal lymph after feeding (Flock et al., 1952). Our failure to find a smallintestinal band in extrahepatic obstruction may be related more directly to the absence of bile from the small intestine, as it is known that some component of bile influences both the character of smallintestinal AP and its level of activity (Cook and Moog, 1970). Thus ligation of the common bile duct in the mouse reduces the AP activity of duodenal homogenates by $\mathbf{4 0 \%}$. It has also been shown that human bile contains a heat-labile stimulator of AP, and that the AP activity of small-intestinal extracts in obstructive jaundice is decreased (Horrigan and Danovitch, 1973). However, neither of these factors serves as a complete explanation for our findings, as, although the extrahepatic group as a whole had a greater degree of obstruction to bile flow as demonstrated by the significantly higher serum bilirubin level (Table 2), many of the patients in the intrahepatic group with a gut band on electrophoresis had a significant degree of obstruction (Fig. 2), possessing higher levels of serum bilirubin than some of the patients in the extrahepatic group, in whom a gut band was never found.

We have recently reported a decreased incidence of gut bands in osteoblastic bone disease, where we postulated that an increased serum contribution from one source may 'switch off' that from the small intestine (Warnes et al., 1976) but this explanation is not applicable to our present findings in liver disease, as the mean AP of the patients with extrahepatic lesions was not significantly higher than that of the patients in the intrahepatic group.

The conclusion of earlier workers was that the determination of serum AP activity is not of value in the diagnosis of hepatic cirrhosis (Gutman, 1959). However, the present study suggests that AP isoenzyme studies play a role, not only in the diagnosis of cirrhosis, but also in differentiating an intrahepatic liver lesion from an extrahepatic cause of jaundice. When no intestinal band is seen, isoenzyme studies do not contribute to this differentiation. However, when a raised serum AP is found in a jaundiced patient and a gut band is present on electrophoresis, the lesion is likely to be intrahepatic.

It is not suggested that AP isoenzyme studies should replace conventional techniques of studying patients with liver disease, but they can provide valuable additional information. In a recent study (Elias et al., 1975) percutaneous transhepatic cholangiography (PCC) failed in $52 \%$ of cases of cholestatic jaundice when employed as the initial procedure, while ERCP failed in $38 \%$. However, in patients with dilated intrahepatic bile ducts, PCC had a $91 \%$ success rate. It would therefore clearly be helpful to know whether the lesion is likely to be intrahepatic or extrahepatic when deciding whether to employ PCC or ERCP as the initial investigation. In those centres which do not specialise in the diagnosis of patients with liver disease, an AP isoenzyme electrophoresis provides a simple, rapid, and nontraumatic screening test which will successfully indicate the need for a conservative non-surgical approach in almost $50 \%$ of cases of intrahepatic jaundice. On the other hand, in more specialised centres which employ both PCC and ERCP, AP isoenzyme studies will help to indicate which investigation should be employed as the procedure of first choice.

We would like to thank Professor H. T. Howat for his support during this project. We thank also Dr R. Ollerenshaw and the Department of Medical Illustration, Manchester Royal Infirmary, for preparation of the photograph, Mrs C. Howland of the MRC Statistics Unit, Gower Street, London, for statistical assistance; and Mrs D. Riley for secretarial help.

\section{References}

Cook, R., and Moog, F. (1970). Influence of biliary stasis 
on alkaline phosphatase in the small intestine. Biochimica et Biophysica Acta, 215, 220 -223.

Cotton, P. B. (1972). Cannulation of the papilla of Vater by endoscopy and retrograde cholangiopancreatography (E.R.C.P.). Gut, 13, 1014-1025.

Elias, E., Hamlyn, A. N., Jain, S., Long, R., Summerfield, J. A., Dick, R., and Sherlock, S. (1975). A randomized trial of percutaneous transhepatic cholangiography versus endoscopic retrograde cholangiography for bile duct visualization in cholestasis. (Abstract) Gut, 16, 831 .

Evans, J. A., Glenn, F., Thorbjarnarson, B., and Mujahed, Z. (1962). Percutaneous transhepatic cholangiography. Radio$\log y, 78,362-370$.

Flock, E. V., Block, M. A., Bollman, J. L., and Mann, F. C. (1952). Alkaline phosphate and amylase of plasma after hepatectomy. American Journal of Physiology, 170, 467-471.

Fishman, W. H., Inglis, N. I., and Krant, M. J. (1965). Serum alkaline phosphatase of intestinal origin in patients with cancer and with cirrhosis of the liver. Clinica Chimica Acta, 12, 298-303.

Gutman, A. B. (1959). Serum alkaline phosphatase activity in diseases of the skeletal and hepatobiliary systems. A consideration of the current status. American Journal of Medicine, 27, 875-901.

Horrigan, F. D., and Danovitch, S. H. (1973). Human jejunal alkaline phosphate activity (AP) in mucosal specimens from normals and a patient with obstructive jaundice. (Abstract) Gastroenterology, 64, 748.

James, M. (1971). Normal or 'negative' percutaneous cholangiogram. Archives of Surgery, 103, 31-33.

Kind, P. R. N., and King, E. J. (1954). Estimation of plasma phosphatase by determination of hydrolysed phenol with amino-antipyrine. Journal of Clinical Pathology, 7, 322-326.

Langman, M. J. S., Leuthold, E., Robson, E. B., Harris, J., Luffman, J. E., and Harris, H. (1966). Influence of diet on the 'intestinal' component of serum alkaline phosphatase in people of different $\mathrm{ABO}$ blood groups and secretor status. Nature, 212, 41-43.

Okuda, K., Tanikawa, K., Emura, T., Kuratomi, S., Jinnouchi, S., Urabe, K., Sumikoshi, T., Kanda, Y., Fukuyama, Y., Musha, H., Mori, H., Shimokawa, Y., Yakushiji, F., and Matsuura, Y. (1974). Nonsurgical, percutaneous transhepatic cholangiography: diagnostic significance in medical problems of the liver. American Journal of Digestive Diseases, 19, 21-36.

Powell, W. N. (1944). Method for the quantitative determination of serum bilirubin with photoelectric colorimeter. American Journal of Clinical Pathology, 8, 55-58.

Roberts, W. M. (1933). Blood phosphatase and the Van den Bergh reaction in the differentiation of the several types of jaundice. British Medical Journal, 1, 734-738.

Schimmel, E. M. (1968). Diagnostic procedures in liver disease. Medical Clinics of North America, 52, 1407-1416.

Sherlock, S. (1975). Diseases of the Liver and Biliary System, 5th edn, pp. 705-719. Blackwell: Oxford.

Smith, I., Lightstone, P. J., and Perry, J. D. (1968). Separation of human tissue alkaline phosphatases by electrophoresis on acrylamide disc gels. Clinica Chimica Acta, 19, 499-505.

Stolbach, L. L., Krant, M. J., Inglis, N. I., and Fishman, W. H. (1967). Correlation of serum L-phenylalaninesensitive alkaline phosphatase, derived from intestine, with the ABO blood group of cirrhotics. Gastroenterology, 52, 819-827.

Warnes, T. W. (1972). Alkaline phosphatase. Gut, 13, 926-937.

Warnes, T. W., Hine, P., and Kay, G. (1976). Polyacrylamide gel disc electrophoresis of alkaline phosphatase isoenzymes in bone and liver disease. Journal of Clinical Pathology, 29 782-787.

Williams, R. (1968). Fourth Symposium on Advanced Medicine, p. 273. Edited by O. Wrong. Pitman Medical: London. 